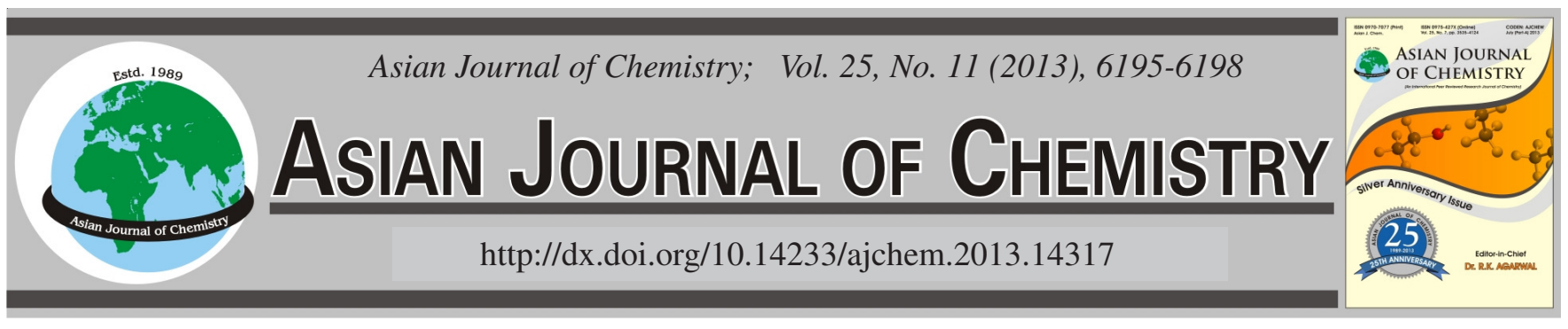

\title{
Synthesis of MA-AM-MMA Copolymer and its Inhibition Performance Against Calcium Phosphate Scale
}

\author{
Aijiang He ${ }^{*}$, Lixiu Liu, Xiangbiao Li and Huiling Yu
}

Department of Biological and Chemical Engineering, Yibin Vocational and Technical College, Xincun Cuipingqu Yibin 644003, Sichuan, P.R. China *Corresponding author: E-mail: scu2000@gmail.com; scu2000@qq.com

\begin{abstract}
A multifunctional ternary copolymer was synthesized from maleic anhydride (MA), acrylamide (AM) and methyl methacrylate (MMA). The effects of the reaction temperature, polymerization time, amount of initiator and monomer ratio on the inhibition performance of the copolymer against calcium phosphate scale were determined. The optimum conditions for synthesis are as follows: reaction temperature, $75^{\circ} \mathrm{C}$; polymerization time, $4 \mathrm{~h}$; amount of initiator, $15 \%$ and monomer molar ratio of MA:AM:MMA, 3:2:1. A scale inhibition efficiency of over $90 \%$ was obtained with the dosage of $25 \mathrm{mg} / \mathrm{L}$ of the copolymer and the transmittance of dispersed ferric oxide was less than 30 $\%$ with the concentration of $10 \mathrm{mg} / \mathrm{L}$ copolymer, which enabled good performance in scale-formation inhibition and dispersion. Infrared spectroscopy results suggested the presence of functional groups, including carboxyl, ester and amide groups, in the terpolymer.
\end{abstract}

Key Words: Maleic anhydride, Acrylamide, Methyl methacrylate, Scale inhibitor, Calcium phosphate.

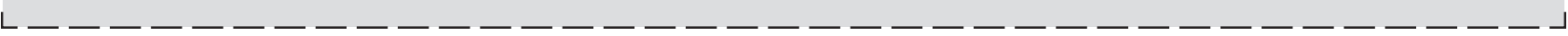

\section{INTRODUCTION}

Alkaline treatment is commonly used in industrial circulating cooling water systems to inhibit corrosion. Therefore, scaling is the major problem that affects the normal operation of these systems. Phosphorus-containing inhibitors of scale formation are the most commonly used water treatment agents. These inhibitors can easily produce phosphate ions and induce the deposition of calcium phosphate $\left[\mathrm{Ca}_{3}\left(\mathrm{PO}_{4}\right)_{2}\right]$ under hydrolysis and microbial action. The solubility of calcium phosphate is extremely low and decreases with increased temperature, making it a "stubborn scale" in water treatments ${ }^{1-3}$.

A copolymer scale inhibitor is a new type of water treatment agent developed after organic phosphate. This inhibitor can effectively prevent the deposition of calcium phosphate, calcium carbonate, calcium sulfate, zinc scale, iron salts, etc. ${ }^{4}$. Thus, cooling water systems can function well under relatively high $\mathrm{pH}$ conditions. In-depth studies on the copolymer structure and mechanism of scale-formation inhibition have revealed a close correlation between the copolymer structure and scale inhibition mechanism. The carboxyl $(-\mathrm{COOH})$, hydroxyl $(-\mathrm{OH})$, acylamino $\left(-\mathrm{CONH}_{2}\right)$ and sulfo $\left(-\mathrm{SO}_{3} \mathrm{H}\right)$ groups in the copolymer all inhibit calcium phosphate scale formation ${ }^{5-7}$.

Maleic anhydride (MA), acrylamide (AM) and methyl methacrylate (MMA) were used as monomers to investigate the performance of the copolymer in inhibiting calcium phosphate scale formation under different synthesis conditions.

\section{EXPERIMENTAL}

Maleic anhydride, acrylamide, methyl methacrylate, ammonium persulfate, ethanol, etc., were analytically pure and purchased from the Chengdu Kelong Company. The equipment were a Nicolet380 FT-IR spectrometer and a self-made thermostatic polymerization reactor consisting of a thermostatic water bath $\left( \pm 0.5^{\circ} \mathrm{C}\right.$ precision $)$, four-necked flask, reflux condenser and a dropping funnel ${ }^{8}$.

Preparation of copolymer: The water bath was kept at a constant temperature of $65^{\circ} \mathrm{C}$. A certain amount of maleic anhydride (MA) was weighed and dissolved in a four-necked flask using distilled water. Methyl methacrylate (MMA), acrylamide (AM) solution and ammonium persulfate solution were sequentially added to three dropping funnels.

At the start of polymerization, an acrylamide solution was added dropwise continuously, with alternating dropwise additions of ammonium persulfate and methyl methacrylate solutions. The addition of these three solutions was finished within $1 \mathrm{~h}$. Then, the temperature was increased for polymerization and kept stable for a period of time. After reaction completion, the products were collected, cooled and filtered under atmospheric pressure. The filtrate was mixed well with ethanol at a ratio of 1:4. The mixture was adjusted to $\mathrm{pH} 7$ with $40 \% \mathrm{NaOH}$ solution, left to stand and cooled for $10 \mathrm{~h}$. The supernatant was decanted and the solid product was oven dried at $110^{\circ} \mathrm{C}$ for $24 \mathrm{~h}$ to obtain the copolymer scale inhibitor. 
Infrared spectrometry: A small amount of washed copolymer was subjected to vacuum drying to achieve a constant weight. The infrared spectrum of the copolymer was measured by the $\mathrm{KBr}$ pellet method using the Nicolet 380 FT-IR spectrometer.

Scale inhibition performance: The inhibition performance of the copolymers against calcium phosphate scale and iron oxide dispersion was examined according to a previous $\operatorname{method}^{9,10}$.

The static scale inhibition method was used to investigate the inhibition performance of the copolymers. For calcium ions in water samples, $\mathrm{C}\left(\mathrm{Ca}^{2+}\right)=250 \mathrm{mg} / \mathrm{L}$ (calculated in $\left.\mathrm{CaCO}_{3}\right)$; for phosphate ions, $\mathrm{C}\left(\mathrm{PO}_{4}{ }^{3-}\right)=50.0 \mathrm{mg} / \mathrm{L}$ and the $\mathrm{pH}$ was adjusted to 9 with $1 \mathrm{~g} / \mathrm{L}$ sodium tetraborate solution. The sample was stored under $80^{\circ} \mathrm{C}$ for $10 \mathrm{~h}$. The supernatant was collected to measure the phosphate ion concentration using bismuth phosphorus molybdenum blue. The inhibition efficiency against $\mathrm{Ca}_{3}\left(\mathrm{PO}_{4}\right)_{2}$ was calculated as $^{11}$ :

$$
\eta=\frac{\mathrm{C}_{2}-\mathrm{C}_{0}}{\mathrm{C}_{1}-\mathrm{C}_{0}} \times 100 \%
$$

where $\mathrm{C}_{0}$ is the phosphate ion concentration in the sample without added copolymer after heating at a constant temperature for $10 \mathrm{~h}(\mathrm{mg} / \mathrm{L}), \mathrm{C}_{1}$ is the phosphate ion concentration in the sample without added copolymer and heating $(\mathrm{mg} / \mathrm{L})$ and $\mathrm{C}_{2}$ is the phosphate ion concentration in the sample with added copolymer and heating at a constant temperature for $10 \mathrm{~h}$ (mg/L).

\section{RESULTS AND DISCUSSION}

IR spectra of synthetic products: The IR spectra in Fig. 1 were obtained from the infrared analysis of the products.

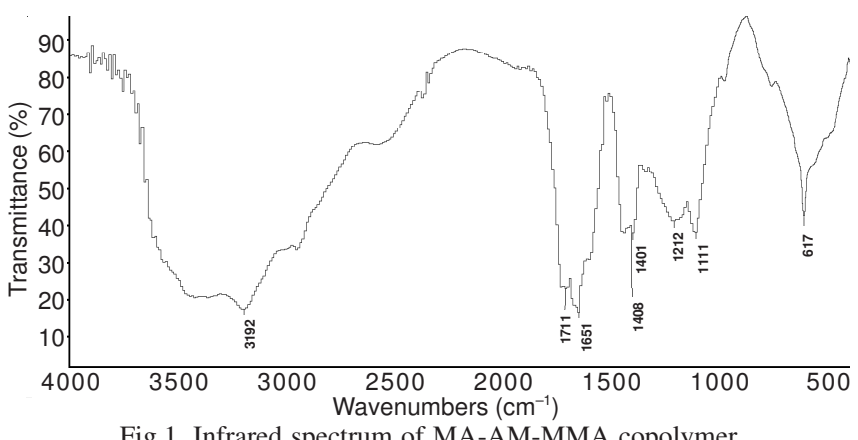

Fig.1. Infrared spectrum of MA-AM-MMA copolymer

Several major characteristic absorption peaks can be seen. The $\mathrm{C}=\mathrm{O}, \mathrm{C}-\mathrm{N}$ and $\mathrm{N}-\mathrm{H}$ stretching vibrations of the amide group were at 1652,1401 and $3193 \mathrm{~cm}^{-1}$, respectively ${ }^{12}$. The $\mathrm{C}=\mathrm{O}$ stretching vibration of the carboxyl group was at 1711 $\mathrm{cm}^{-1}$. The O-H stretching vibration of the carboxyl group and $\mathrm{N}-\mathrm{H}$ stretching vibration of the amide group resulted in a mild absorption peak at $3193 \mathrm{~cm}^{-1}{ }^{13}$. The C-O stretching vibration of the ester group was at $1212 \mathrm{~cm}^{-1}$. These characteristic peaks suggested that the copolymer produced from the polymerization of monomers MA, AM and MMA contained carboxyl, ester and amide groups in the presence of potassium persulfate as the initiator.

Synthesis conditions: The performance of copolymers is related to the type, quantity and spatial distribution of functional groups, as well as to the molecular weight of the copolymer, which is rather small when used as a scale inhibitor. The scale inhibition performance is particularly high when the molecular weight is 1000 to $10000^{14}$.

The test for inhibiting calcium phosphate scale formation was performed using $25 \mathrm{mg} / \mathrm{L}$ copolymers synthesized under different conditions. The inhibition performance of the copolymer was evaluated using the scale inhibition efficiency as an indicator.

Synthesis temperature: Fig. 2 shows the relationship between the synthesis temperature and scale inhibition efficiency at a MA:AM:MMA molar ratio of 1:1:1, initiator accounting for $18 \%$ of the total monomer amount and reaction time of $5 \mathrm{~h}$.

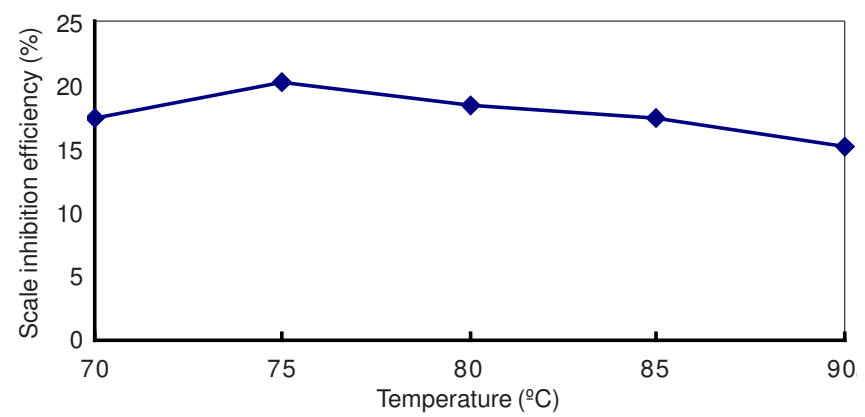

Fig. 2. Influence of synthesis temperature on scale inhibition efficiency

The molecular weight of the copolymer produced decreased with increased temperature. Free-radical polymerization was also accelerated and the chain transfer of the solvent was enhanced. The scale inhibition efficiency reached the maximum at $75^{\circ} \mathrm{C}$. The reaction temperature was controlled at $75^{\circ} \mathrm{C}$ because the scale inhibition efficiency can be adversely affected by the low molecular weight of the polymer with continued temperature increase. The increase in temperature accelerated the chain termination and enhanced the chain branching reaction.

Initiator amount: The amount of initiator was varied to investigate its influence on the scale-formation inhibitory performance of the polymer. The temperature was $75^{\circ} \mathrm{C}$ and the other conditions were unchanged. The results are shown in Fig. 3.

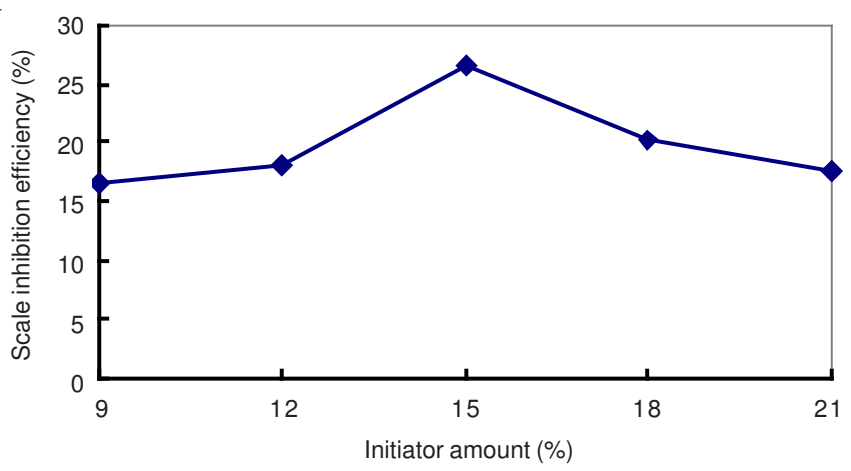

Fig.3. Influence of the initiator amount on the scale inhibition efficiency

Fig. 3 shows that the inhibition efficiency gradually increased with increased initiator amount, reached the maximum 
when the initiator-to-monomer weight ratio was $15 \%$ and then decreased. With increased initiator amount, the relative molecular weight of the polymer decreased, which contributed to the scale dispersion performance of the polymer. However, the initiator amount was too high at this moment and the short molecular chain of the polymer affected the inhibition of calcium phosphate scale formation due to the small number of active sites. This phenomenon increased the synthesis cost, led to unsafe factors and reduced the scale inhibition performance of the polymer. Therefore, the optimum initiator-tomonomer mass ratio was $15 \%$.

Monomer ratio: Fig. 4 shows the relationship between the monomer ratio and scale inhibition performance of the polymer under the optimal reaction temperature and initiator amount.

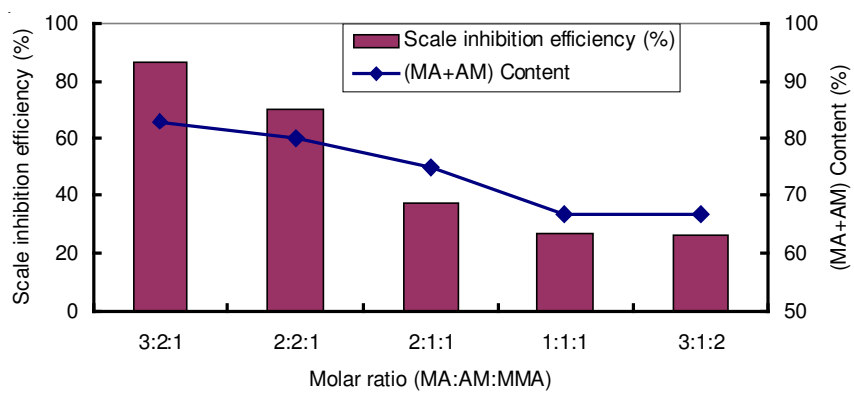

Fig. 4. Influence of the monomer ratio on the scale inhibition efficiency

Fig. 4 shows that the monomer ratio significantly influenced the scale inhibition efficiency of the polymer. The scale inhibition efficiency was $92.5 \%$ and $26.7 \%$ when the MA:AM:MMA ratios were 3:2:1 and 3:1:2, respectively. With increased percentage of the monomers AM and MA, the scale inhibition efficiency also increased.

The amide $\left(-\mathrm{CONH}_{2}\right)$ and carboxyl $(-\mathrm{COOH})$ groups in the polymer are the functional groups responsible for inhibiting calcium phosphate scale formation. There are carboxyl groups in both MA and MMA. However, for the same mass of MA and MMA, the carboxyl group (-COOH) content was higher in MA than in MMA. A higher MA content in the copolymer resulted in better inhibition performance.

Reaction time: Fig. 5 shows the relationship between the polymerization time and scale inhibition performance of the polymer under optimal reaction temperature, initiator amount and monomer ratio.

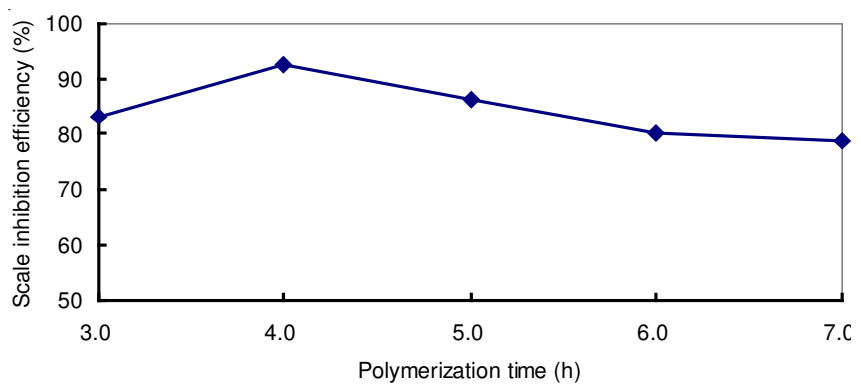

Fig. 5. Influence of the reaction time on the scale inhibition efficiency

The scale inhibition efficiency of the copolymer increased with increased polymerization time, reaching the maximum at a reaction time of $4 \mathrm{~h}$. A longer reaction time resulted in a higher conversion ratio of the monomers and a higher level of polymerization completion. However, an excessively long reaction time resulted in a longer polymer chain, which affected the complexation with metal ions and reduced the inhibition efficiency. Therefore, the optimum polymerization timewas $4 \mathrm{~h}$.

Scale inhibition performance: The scale inhibition performance of the scale inhibitor is associated with the scale inhibitor amount. Calcium ions with concentrations higher than the stoichiometric ratio can be stabilized in water by adding a small amount of scale inhibitor.

Fig. 6 shows that a copolymer amount of a few $\mathrm{m} / \mathrm{gL}$ was sufficient to achieve the scale inhibition effect and to exert a "threshold effect" specific to the scale inhibitor. The scale inhibition efficiency was over $90 \%$ when the copolymer amount was $25 \mathrm{mg} / \mathrm{L}$, which indicated that the synthesized copolymer well inhibited calcium phosphate scale formation.

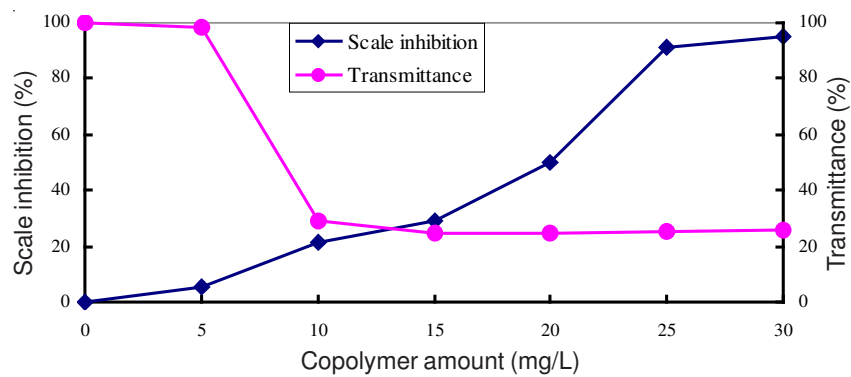

Fig. 6. Effect of copolymer concentration on scale inhibition performance and dispersion ability

Dispersion ability: The dispersion ability is another important indicator of scale inhibitor, especially iron oxide dispersion, which is as an important component of anti-corrosion measures. Cooling water systems contain certain amounts of iron oxides and dissolved iron can induce the deposition of phosphate ions, thereby affecting the scale inhibition performance of the inhibitor. Iron oxides hydrolyzed from $\mathrm{FeSO}_{4} \cdot 7 \mathrm{H}_{2} \mathrm{O}$ at high $\mathrm{pH}$ values act as a suspension medium. The ability of the scale inhibitor to disperse and inhibit iron oxide deposition under static conditions is evaluated by changing the scale inhibitor concentration.

Fig. 6 shows that the transmittance almost remained constant when the copolymer amount was less than $5 \mathrm{mg} / \mathrm{L}$. The transmittance rapidly decreased with increased addition amount, which suggested that the amount of iron ions dispersed in the solution increased. By continuously increasing the copolymer amount, the transmittance slightly changed with a copolymer concentration of $10 \mathrm{mg} / \mathrm{L}$ and the transmittance decreased to $29.2 \%$. The copolymer was proved to have good dispersion performance and effectively dispersed ferric oxide at low concentrations.

\section{Conclusion}

A terpolymer was synthesized using maleic anhydride, acrylamide and methyl methacrylate as monomers, as well as ammonium persulfate as the initiator. The optimum synthesis conditions were as follows: monomer molar ratio MA:AM:MMA, $3: 2: 1$; initiator amount, $15 \%$; reaction temperature, $75^{\circ} \mathrm{C}$ and 
polymerization time, $4 \mathrm{~h}$. Infrared spectroscopy results suggested that carboxyl, ester and amide groups were present in the synthesized terpolymer.

The scale-formation inhibition performance of the polymer was most significantly influenced by the monomer ratio. Higher MA and MMA contents resulted in better scale inhibition performance. When the added amount of copolymer was $25 \mathrm{mg} / \mathrm{L}$, the scale inhibition efficiency exceeded $90 \%$ and the copolymer well inhibited calcium phosphate scale formation. The scale inhibitor was also proved to have good dispersion performance. When the added amount was over $10 \mathrm{mg} / \mathrm{L}$, ferric oxide was effectively dispersed and stabilized in water.

\section{REFERENCES}

1. Z. Amjad, The Science and Technology of Industrial Water Treatment, CRC Press (2009).

2. C.Y. Jianping, Chem. Ind. Times, 22, 27 (2008)
3. L. Perez and S. Kessler, Water Soluble Polym., 214, 149 (2002).

4. J.K. Fink, Petroleum Engineer's Guide to Oil Field Chemicals and Fluids, Gulf Prof. Publ., Boston, pp. 253-274 (2012).

5. Y. Sun, X. Zhou and L. Ma, J. Dalian Railway Inst., 25, 93 (2004).

6. P. Shakkthivel and T. Vasudevan, Desalination, 197, 179 (2006).

7. Y. Zhang J. Wu and S. Hao, Chin. J. Chem. Eng., 15, 600 (2007).

8. L.L. Liu, A.J. He and X.B. Li, Asian J. Chem., 24, 2298 (2012).

9. B. Smith, Desalination, 3, 263 (1967).

10. Y. Xu, L.L. Zhao and L.N. Wang, Desalination, 286, 285 (2011).

11. R. Pairat, C. Sumeath and F.H. Browing, Langmuir, 13, 1791 (1997).

12. R. Touir, N. Dkhireche and M.E. Touhami, Desalination, 249, 922 (2009).

13. R. Touir, M. Cenoui and M. Elbakri, Corros. Sci., 50, 1530 (2008).

14. Z. Amjad, J. Colloid Interf. Sci., 123, 523 (1988). 